\title{
A new CARD15 mutation in Blau syndrome
}

\author{
Marjan M van Duist ${ }^{1}$, Mario Albrecht ${ }^{2}$, Marta Podswiadek ${ }^{3}$, Daniela Giachino ${ }^{1}$, \\ Thomas Lengauer ${ }^{2}$, Leonardo Punzi ${ }^{3}$ and Mario De Marchi ${ }^{*, 1}$
}

\author{
${ }^{1}$ Department of Clinical and Biological Sciences, Division of Medical Genetics, University of Torino, Orbassano, Italy; \\ ${ }^{2}$ Department of Computational Biology and Applied Algorithmics, Max Planck Institute for Informatics, Saarbrücken, \\ Germany; ${ }^{3}$ Division of Rheumatology, Department of Medical and Surgical Sciences, University of Padova, Padova, \\ Italy
}

The caspase recruitment domain gene CARD15/NOD2, encoding a cellular receptor involved in an NF- $\kappa$ Bmediated pathway of innate immunity, was first identified as a major susceptibility gene for Crohn's disease (CD), and more recently, as responsible for Blau syndrome (BS), a rare autosomal-dominant trait characterized by arthritis, uveitis, skin rash and granulomatous inflammation. While CARD15 variants associated with $C D$ are located within or near the C-terminal leucine-rich repeat domain and cause decreased NF- $\kappa$ B activation, BS mutations affect the central nucleotide-binding NACHT domain and result in increased NF- $\kappa$ B activation. In an Italian family with BS, we detected a novel mutation E383K, whose pathogenicity is strongly supported by cosegregation with the disease in the family and absence in controls, and by the evolutionary conservation and structural role of the affected glutamate close to the Walker B motif of the nucleotide-binding site in the NACHT domain. Interestingly, substitutions at corresponding positions in another NACHT family member cause similar autoinflammatory phenotypes. European Journal of Human Genetics (2005) 13, 742-747. doi:10.1038/sj.ejhg.5201404

Published online 6 April 2005

Keywords: Blau syndrome; CARD15; NOD2; NACHT; activating mutation

\section{Introduction}

Blau syndrome (BS, MIM 186580) is a rare Mendelian trait with autosomal-dominant inheritance characterized by multiorgan granulomatous inflammation of the skin, eyes and joints. ${ }^{1}$ The BS locus was mapped by Tromp et al ${ }^{2}$ to the chromosomal region 16q12.1-13, which also contains one of several inflammatory bowel disease (IBD) susceptibility loci. ${ }^{3-5}$ The caspase recruitment domain gene CARD15, previously named NOD2, was subsequently identified in that region both as a major determinant of susceptibility to Crohn's disease (CD) ${ }^{6-8}$ and as the gene responsible for BS. ${ }^{9}$ The encoded multidomain protein of 1040 amino acids is implicated in an

${ }^{*}$ Correspondence: Professor M De Marchi, Department of Clinical and Biological Sciences, Division of Medical Genetics, University of Torino, Regione Gonzole 10, Orbassano 10043, Italy.

Tel: + 39011670 5425/930 8611; Fax: + 39011903 8639;

E-mail: mario.demarchi@unito.it

Received 30 November 2004; revised 4 February 2005; accepted 8 February 2005
NF- $\kappa$ B-mediated pathway of inflammation and apoptosis and is mainly expressed in monocytes, granulocytes and dendritic cells, which are major components of BS granulomas. CDassociated variants mostly affect the C-terminal protein domain consisting of leucine-rich repeats (LRRs) and cause decreased NF- $\kappa$ B signaling. ${ }^{10,11}$ In contrast, mutations up to now identified in BS patients are missense changes affecting residues 334 and 469 of the nucleotide-binding domain (NBD) and have been shown to result in increased basal NF- $\kappa \mathrm{B}$ activity, consistent with the dominant inheritance of the syndrome. ${ }^{10,11}$ Here, we describe a different mutation that substitutes glutamate E383 adjacent to the $\mathrm{Mg}^{2+}$-binding (Walker B) motif with lysine. Our finding extends the mutational spectrum of CARD15 in BS and contributes to a better understanding of its pathogenesis in the context of a common molecular mechanism shared with other evolutionarily related proteins containing nucleotide-binding and LRR domains involved in human inflammatory disorders ${ }^{12-14}$ and plant disease resistance. ${ }^{15,16}$ 


\section{Patients and methods}

The proband (II4 in Figure 1), now 51 years, was first seen at the age of 10 years and diagnosed on the basis of described criteria for BS, ${ }^{1,17}$ including typical ultrastructural findings in dermal and synovium granulomas. Main signs were skin rash at the extensory aspect of upper and

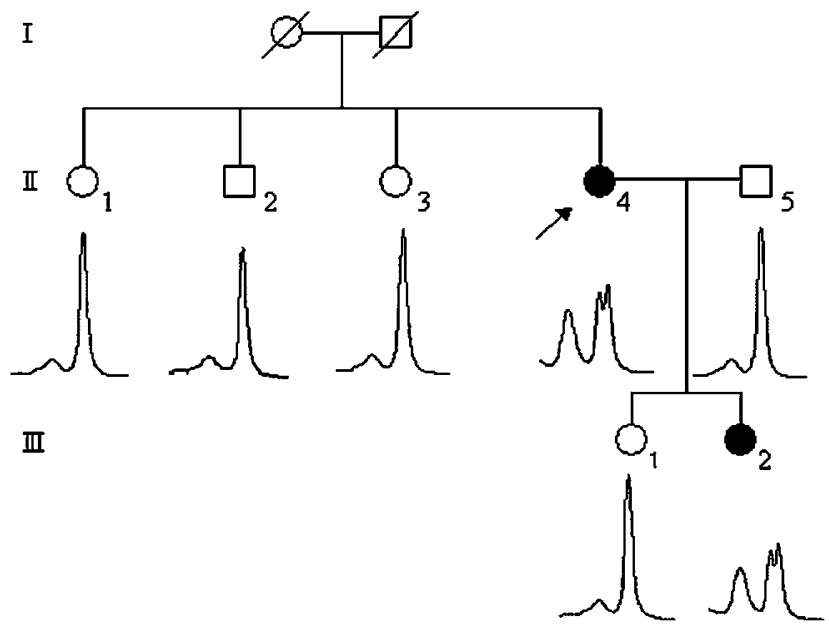

Figure 1 Pedigree of the family. Subjects II4 and III2 are affected with BS. DHPLC shows a clear difference between the three-peak pattern of affected E383K heterozygotes and the single-peak pattern of healthy family members. lower limbs, arthritis of the small hand joints and severe bilateral uveitis. The daughter (III2) showed similar skin rash and arthritis since the age of 5 years without ocular involvement. Sibs II1, II2, II3 and the other daughter (III1) showed no symptoms of BS. Both patients and the relatives indicated in Figure 1 gave written consent to genetic investigation on the CARD15 or other candidate BS genes. DNAs of 100 healthy medical students belonging to the Italian population and enrolled according to the rules of our institution were used as normal population controls.

Coding sequences of the CARD15 gene (exons 2-11, NCBI accession number NM_022162) were amplified from genomic DNA, using the primers and polymerase chain reaction (PCR) conditions shown in Table 1. Amplified products were size controlled by electrophoresis on $2 \%$ agarose, directly sequenced in the two affected subjects and analyzed in all family members by denaturing highperformance liquid chromatography (DHPLC) using the Wave system (Transgenomic). The three CD-associated variants R702W, G908R and L1007fs were genotyped by restriction analysis of exons 4,8 and 11 as described previously. ${ }^{18}$

We used the PSI-BLAST suite ${ }^{19}$ to search for homologs (E-value cutoff 0.005 ) in the UniProt database. ${ }^{20}$ Multiple sequence alignments were assembled by $\mathrm{T}^{-C_{0}} \mathrm{CFFE}^{21}$ and improved manually by minor adjustments. Protein structures and secondary structure assignments were obtained

Table 1 CARD15 primers, PCR and DHPLC conditions of exons 2-11

\begin{tabular}{|c|c|c|c|c|}
\hline Exon & Domain & Sense $(F)$; reverse $(R)$ & $T P C R$ & $T D H P L C$ \\
\hline 2 & CARD $1+2$ & $\begin{array}{l}\text { (F) 5'-GCATCTGGCTTCTGGAGAAGT } \\
\text { (R) 5'-ACCCCTTTCCTGAGAACTCTG }\end{array}$ & 61 & 63.5 \\
\hline 3 & - & $\begin{array}{l}\text { (F) 5'-CCATCAGCCTTCCTGGAAG } \\
\text { (R) 5'-CATGGATCTGCACTGACTGC }\end{array}$ & 57 & 59.8 \\
\hline $4 a$ & NBD & $\begin{array}{l}\text { (F) 5'-GGCTGGCTCTCCTATCCCTT } \\
\text { (R) 5'-TTGACCAACATCAGGCCAACAG }\end{array}$ & 61 & $61.5-63.5$ \\
\hline $4 b$ & NBD & $\begin{array}{l}\text { (F) 5'-CTGCAGGGGAAGACTTCCA } \\
\text { (R) 5'-AGGGCTGAGGTCTCTTGGA }\end{array}$ & 61 & $61.1-63.1$ \\
\hline $4 c$ & NBD & $\begin{array}{l}\text { (F) 5'-TCCGCACCGAGTTCAACCTC } \\
\text { (R) 5'-CGCGGCAAAGAAGCACTGGA }\end{array}$ & 61 & $62.4-63.2$ \\
\hline $4 d$ & - & $\begin{array}{l}\text { (F) 5'-GCCAAGAGCGTGCATGCCAT } \\
\text { (R) 5'-CACCAGACCCAGCACATAGG }\end{array}$ & 61 & 62.2 \\
\hline 5 & LRR & $\begin{array}{l}\text { (F) 5'-GGAAGCACAGATGCTGGCAC } \\
\text { (R) 5'-TGAGACCAGGGCACTTGGAG }\end{array}$ & 62 & $57.0-61.0$ \\
\hline 6 & LRR & $\begin{array}{l}\text { (F) 5'-GGTGCTCACTGTCCAATGTGC } \\
\text { (R) 5'-GACTGACTCAGGAATGGGCC }\end{array}$ & 57 & $59.6-60.6$ \\
\hline 7 & LRR & $\begin{array}{l}\text { (F) 5'-CGCTGTGTTCTCTCAGCCT } \\
\text { (R) 5'-CTGAAGAGTTTCACCTGCCC }\end{array}$ & 58 & $62.0-65.0$ \\
\hline 8 & LRR & $\begin{array}{l}\text { (F) 5'-CACTGACACTGTCTGTTGACTC } \\
\text { (R) 5'-AAGACCTTCATAAGTGGCCCC }\end{array}$ & 60 & $61.3-63.3$ \\
\hline 9 & LRR & $\begin{array}{l}\text { (F) 5'-GGTTAGCTCATCTCTCGAGGTC } \\
\text { (R) 5'-GATTGTGATCCACTTCCCCAG }\end{array}$ & 59 & 60.2 \\
\hline 10 & LRR & $\begin{array}{l}\text { (F) 5'-GGGGCATGTGAGTTCATCATC } \\
\text { (R) 5'-GCGGCCCAGATCTTAATCAG }\end{array}$ & 59 & $58.3-59.3$ \\
\hline 11 & LRR & $\begin{array}{l}\text { (F) 5'-CTGGCTAACTCCTGCAGT } \\
\text { (R) 5'-ACTGAGGTTCGGAGAGCT }\end{array}$ & 58 & $58.9-61.4$ \\
\hline
\end{tabular}

Products amplified with a standard PCR protocol using the indicated sense $(\mathrm{F})$ and reverse $(\mathrm{R})$ primers at the annealing temperature indicated in column 'T PCR' and analyzed in DHPLC at the elution temperature indicated under 'T DHPLC'. 
from the PDB and DSSP databases, ${ }^{22,23}$ and the web servers PSIPRED, SAM-T99 and SSpro2 predicted the secondary structure of proteins to form a consensus prediction by majority voting. ${ }^{24}$ The sequence alignments depicted in Figure 2 were illustrated using the online service ESPript. ${ }^{25}$ UniProt accession numbers and synonymous names of selected proteins are as follows: $\mathrm{F}_{1}$-ATPase, Q9T2U4; I-2, Q9XET3; CARD15/NOD2, Q9HC29; CARD4/NOD1, Q9Y239; PYPAF1/NALP3/CIAS1/Cryopyrin, Q96P20; PYPAF2/NALP2, Q9NX02; PYPAF3/NALP7, Q8WX94; PYPAF4/NALP4, Q96MN2; PYPAF5/NALP6, P59044; PYPAF6/ NALP11, P59045; PYPAF7/NALP12/MONARCH-1, P59046; and PYPAF8/NALP5/MATER, P59047. The PDB identifier of the crystal structure of bovine $\mathrm{F}_{1}$-ATPase is $1 \mathrm{bmf}$, and we used chain $\mathrm{F}$ that corresponds to the $\beta$-subunit.

\section{Results}

Direct sequencing of the PCR-amplified segment of exon 4 encoding the NBD (codons 273-577) of CARD15 in the two affected subjects identified a heterozygous $G \rightarrow A$ substitution at nucleotide 1147, changing glutamate to lysine (E383K). DHPLC analysis confirmed a heteroduplex pattern segregating from the mother to the affected a

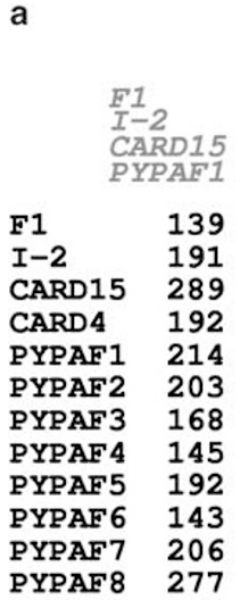

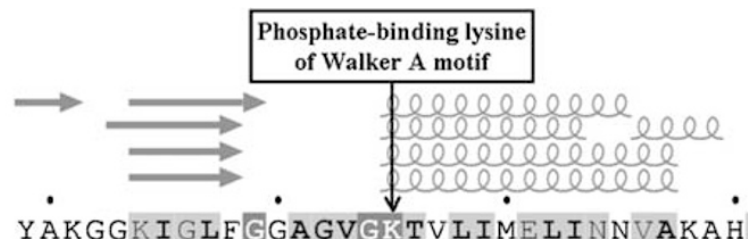
YAKGGKIGLF GGAGVGKTVLIMELINNVAKAH
GKKLTVVPIVGMGGQGKTTLAKAVYNDERVK. NEQGETIFIIT GPFSYTVVLYGPAGLGKT T LAQKLMLDWAEDNLIH. KFKYAFYLSCRELSR KLTPYTVVLHGPAGVGKT T LAKKCMLDWTDCNLSPT . LRYAFYLSCKELSR GKQPRTVI I $Q$ GP G I GKT T LLMKLMMAW DNK IFRDRF LY TFYF CCRELRE GRRP L TVVLQGPAG I GKTMAAKK I I YDWAAGKLYQGQVDFAF F MPC GEL LE SANN LNVE LMGERA S GKT I VIN LAVLRW I KG EMWQNM I SYVVH L T S HE INQ P EP P RTVVMQGAAG I GKSMLAHKVMLDWAD GKLF Q GRF DY LFY INCREMNQ GFRPRTVVLHGK S G IGKSA LARR IVLCWAQG GLYQGMF SYVFF LP VREMQR

b

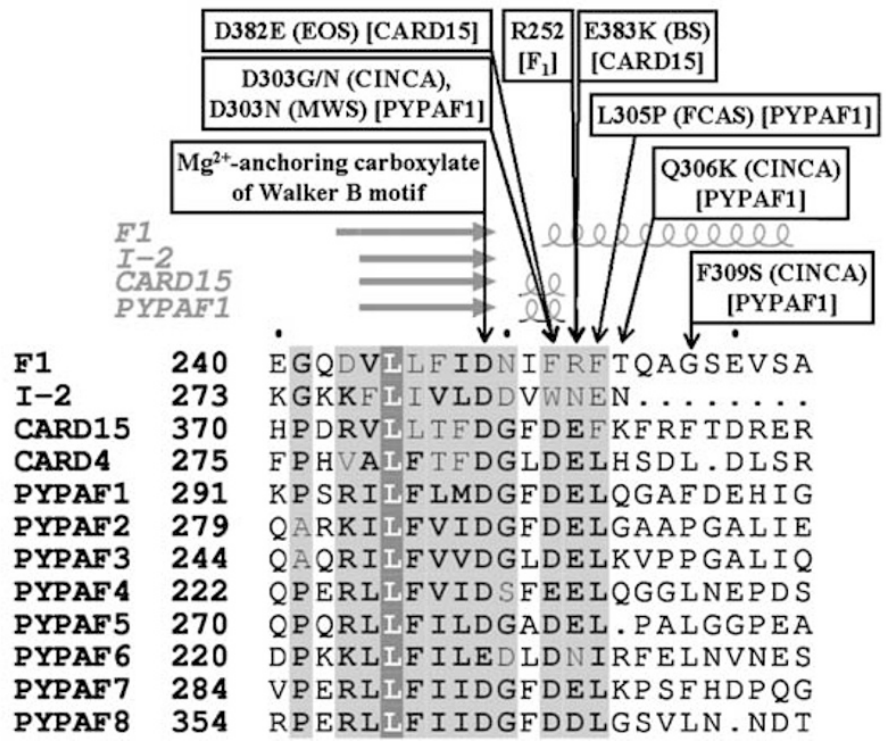

Figure 2 Structure-based multiple sequence alignment of nucleotide-binding sites in NACHT ATPase domains of human CARD15/4 and PYPAF1-8, in the ATPase domain of tomato disease resistance protein I-2 and in the $\beta$-subunit of bovine $\mathrm{F}_{1}$-ATPase. (a) Sequence region with Walker A motif including the phosphate-binding lysine. (b) Sequence region with Walker B motif including the $\mathrm{Mg}^{2+}$-anchoring aspartate. The secondary structure of $\mathrm{F}_{1}$-ATPase and the corresponding consensus predictions for I-2, PYPAF1 and CARD15 are depicted in the upper part ( $\alpha$-helices as curled lines, $\beta$-strands as horizontal arrows). Alignment columns with strictly conserved residues are highlighted in dark gray boxes, and those in which more than $60 \%$ of all residues are physicochemically equivalent are shown in light gray boxes. Text labels point to functionally relevant residues and disease-associated missense variants. 
daughter and to none of the healthy sibs (Figure 1). This variant pattern was not found in any of 100 controls. Sequencing and DHPLC analysis of the entire CARD15 coding sequence in the affected subjects did not detect any other variant of probable pathogenic significance. Typing by restriction analysis excluded the presence of the three recurrent $\mathrm{CD}$-associated variants in the family.

A PSI-BLAST search was performed with residues from 273 to 577 of the CARD15 gene product against the UniProt database. This search returned many members of the NACHT domain subfamily. ${ }^{26,27}$ E383 is conserved in 85 of the 138 sequences (61.6\%) included in the complete NACHT domain sequence alignment of the Pfam database (release 15.0), ${ }^{28}$ which indicates a higher evolutionary conservation than that of other residues mutated in BS: R334, conserved in 67 sequences (48.6\%), and L469, contained in an unconserved loop after the C-terminus of the Pfam NACHT alignment. For the sake of simplicity, we selected the closely related CARD15, CARD4 and PYPAF1-8 proteins containing the CARD and PYD domain, respectively, for further analyses (Figure 2) ${ }^{12-14}$ In addition, we compared these protein sequences with the corresponding segments of more distantly related proteins such as the tomato resistance gene product $\mathrm{I}-2^{29}$ and the bovine $\mathrm{F}_{1}$ ATPase $^{30}$ in order to gain insights on the structural and functional role of mutated residues. A multiple sequence alignment of the selected segments is shown together with the relevant structural motifs around R334 mutated in previous BS cases (Figure 2a) and E383 mutated in this case (Figure 2b).

\section{Discussion}

The CARD15 gene encodes a cellular recognition molecule mediating NF- $\kappa \mathrm{B}$ activation in response to the muramyl dipeptide component of bacterial peptidoglycan. ${ }^{13,31}$ The identification of CARD15 as a major gene involved in susceptibility to $\mathrm{CD}^{6-8}$ opened new insights on deregulated natural immunity in IBD and corroborated the longheld hypothesis that its pathogenesis involves abnormal reaction to intestinal microorganisms by showing that it is caused by the failure of the C-terminal LRR receptor domain to recognize bacterial products. ${ }^{13,31}$ A distinct group of mutations in the same CARD15 gene causes, in heterozygous state, the rare autoinflammatory BS. ${ }^{9,32}$ The Mendelian pattern of inheritance indicates that BS mutations are more penetrant and may affect CARD15 function more severely than the common CD-associated variants. Actually, the latter are thought of as recessive, or genedose-dependent, predisposing factors, with an estimated penetrance $<4 \%$ in homozygotes/compound heterozygotes and even lower in heterozygotes. ${ }^{33}$ Moreover, the inflammatory manifestations of BS affect multiple organs and tissues expressing the CARD15 gene, without any apparent dependence on intestinal stimuli.
Known BS mutations are missense changes in specific positions of the NBD named NACHT, which characterizes a distinct protein subfamily of the STAND NTPases. ${ }^{27}$ In most proteins, the NACHT domain is centrally located between a C-terminal sensor domain (LRRs in CARD15/4 and PYPAF1-8) and an N-terminal adaptor domain (CARD/ PYD). According to current models, the NACHT domain undergoes, in response to a specific ligand-LRR interaction, stereotyped, NTPase-mediated conformational changes that involve homophilic oligomerization, recruitment of downstream factors, and assembly and dissolution of a signaling complex eventually leading to NF- $\kappa \mathrm{B}$ activation. ${ }^{11-14,27,34}$ A similar switch changing between an inactive ADP-bound and an active ATP-hydrolyzing conformation functions in the NACHT-related NBDs of apoptotic protease-activating factor 1 (APAF1), with a WD40 repeat sensor domain and a CARD adaptor domain regulating apoptosome activation, ${ }^{35}$ and of plant disease resistance proteins, activated by pathogen recognition through their LRRs. ${ }^{15,16}$ This regulatory NTPase switch can be compared with that described in detail for other ATPases such as the valosin-containing protein p97, 36,37 a member of the NACHT/STAND-related AAA + family, $^{27}$ and trimeric $G$ proteins. ${ }^{37-39}$ In all cases, NTPase domains are generally assumed to work in signaling cascades and to provide scaffolds for NTP-dependent assembly of protein complexes that integrate signals transmitted by different sensor domains. ${ }^{27,36}$

NACHT domain proteins, besides containing the functionally relevant Walker A and B sequence motifs common in many other nucleotide-binding proteins, are characterized by the presence of two tiny residues downstream of the magnesium binding aspartate, followed by two acid residues. Previously known mutations from BS patients affect arginine R334 and leucine L469 of the NACHT domain. The first is located downstream of the nucleotidebinding Walker A motif. L469 is predicted, on the basis of the crystal structure of the related NBD of $\mathrm{F}_{1}$-ATPase, to be located in the flexible linker downstream of the NACHT domain wrapped around the nucleotide-binding site, and its substitution presumably affects ATP hydrolysis. ${ }^{30,36,37}$ Moreover, a mutation converting aspartate D382 to glutamate has been recently reported in early-onset sarcoidosis (EOS). ${ }^{40}$ In functional assays, all three BS mutations and D382E exhibited enhanced basal NF- $\kappa \mathrm{B}$ activity compared to wild-type CARD15. ${ }^{10,40}$

The new mutation in our patients changes a different residue of the NACHT domain, glutamate E383 to lysine. Despite the lack of a direct functional assay, its pathogenicity is strongly supported by several, although indirect, pieces of evidence. First, it clearly cosegregates with the disease in a dominant manner since it is transmitted from the affected mother to the affected daughter and not to the healthy sib. Second, it was not detected in a sample of 100 unrelated individuals, that is, 200 alleles. 
Third, evolutionary conservation within the NACHT domain family $^{27}$ is stronger for E383 than for the BSassociated positions R334 and L469. It should be noted that the evolutionary conservation is not absolute, for instance, an aspartate is present in place of E383 in PYPAF8 of man (Figure 2b) and mouse (not shown), and an arginine in F1ATPase (Figure 2) and human telomerase-associated protein 1 (not shown). However, evolutionary conservation in paralogous proteins cannot always be taken as an absolute criterion of pathogenicity, and the observed conservation already points to the functional importance of the residue E383 in the NBD.

Fourth, structural modeling of the NACHT domain of CARD15 indicates that D382 and E383 are a couple among several acidic residues in the Walker $B$ motif that are involved in anchoring a $\mathrm{Mg}^{2+}$ ion by hydrogen bonds through a coordinated water molecules. ${ }^{36}$ Since E383 is one of the distinguishing features of the NACHT subfamily, $^{27}$ its substitution with a positively charged lysine is expected to alter a fine-tuned, and possibly negative, regulatory mechanism that is specific of it, rather than drastically affect ATP hydrolysis. ${ }^{11,27,36,41}$

Fifth, all three residues affected by BS mutations correspond to the position of pathogenic mutations in the closely related protein PYPAF1 that cause three other autosomal-dominant autoinflammatory diseases, chronic infantile neurological cutaneous and articular syndrome (CINCA), familial cold autoinflammatory syndrome (FCAS) and Muckle-Wells syndrome (MWS). As previously noted, the BS-associated CARD15 mutations R334Q/W correspond to the PYPAF1 mutations R260L/P and R260W associated with CINCA and FCAS/MWS (Figure 2a). ${ }^{13,14,36,41,42}$ This correspondence also holds true for E383 that is located next to D382, which is mutated in EOS, and between the positions of the pathogenic mutations D303G/N and L305P in PYPAF1 (Figure 2b). Like the BS-associated missense changes R334Q/W and L469F, D303N and D382E also cause a substantial increase of basal NF- $\kappa \mathrm{B}$ activity. ${ }^{10,40,43}$ Interestingly, significantly reduced catalytic rates of ATP hydrolysis have been observed experimentally when mutating the corresponding residues E181, R182, E185 and R246 of $F_{1}$-ATPase ${ }^{44,45}$ and S233 of I-2 (Tameling, personal communication).

In conclusion, all BS variants lie close to the ATP-binding site and are predicted to either directly affect ATP hydrolysis or indirectly modulate the conformational switch through side chain movements of adjacent amino acids. This might in turn allow excessive signaling due to a reduced disassembly of CARD15 signaling complexes, and result in constitutively increased NF- $\kappa$ B activation, even in the absence of the TLR2-mediated bacterial stimuli that occur in the intestine and have recently been implicated in the pathogenesis of CD. ${ }^{46}$ Owing to the common inflammatory pathophysiology of patients with BS and MWS, as well as the apparent functional similarities between
CARD15 and PYPAF1, treatment with an interleukin- $1 \beta$ antagonist, which has been successful for FCAS and MWS ${ }^{47,48}$ might also be proposed for BS patients.

\section{Acknowledgements}

We thank Iris Antes, Francisco $S$ Domingues and Wladimir IL Tameling for helpful discussions. This work was supported in part by grants of the University of Torino and the German Research Foundation (DFG) under contract number LE 491/14-1.

\section{References}

1 Blau EB: Familial granulomatous arthritis, iritis, and rash. J Pediatr 1985; 107: 689-693.

2 Tromp G, Kuivaniemi H, Raphael S et al: Genetic linkage of familial granulomatous inflammatory arthritis, skin rash, and uveitis to chromosome 16. Am J Hum Genet 1996; 59: 1097-1107.

3 Hugot JP, Laurent-Puig P, Gower-Rousseau C et al: Mapping of a susceptibility locus for Crohn's disease on chromosome 16. Nature 1996; 379: 821-823.

4 Ohmen JD, Yang HY, Yamamoto KK et al: Susceptibility locus for inflammatory bowel disease on chromosome 16 has a role in Crohn's disease, but not in ulcerative colitis. Hum Mol Genet 1996; 5: $1679-1683$.

5 Curran ME, Lau KF, Hampe J et al: Genetic analysis of inflammatory bowel disease in a large European cohort supports linkage to chromosomes 12 and 16. Gastroenterology 1998; 115: $1066-1071$

6 Hampe J, Cuthbert A, Croucher PJ et al: Association between insertion mutation in NOD2 gene and Crohn's disease in German and British populations. Lancet 2001; 357: 1925-1928.

7 Hugot JP, Chamaillard M, Zouali H et al: Association of NOD2 leucine-rich repeat variants with susceptibility to Crohn's disease. Nature 2001; 411: 599-603.

8 Ogura $\mathrm{Y}$, Bonen DK, Inohara $\mathrm{N}$ et al: A frameshift mutation in NOD2 associated with susceptibility to Crohn's disease. Nature 2001; 411: 603-606.

9 Miceli-Richard C, Lesage S, Rybojad M et al: CARD15 mutations in Blau syndrome. Nat Genet 2001; 29: 19-20.

10 Chamaillard M, Philpott D, Girardin SE et al: Gene-environment interaction modulated by allelic heterogeneity in inflammatory diseases. Proc Natl Acad Sci USA 2003; 100: 3455-3460.

11 Tanabe T, Chamaillard M, Ogura Y et al: Regulatory regions and critical residues of NOD2 involved in muramyl dipeptide recognition. EMBO J 2004; 23: 1587-1597.

12 Chamaillard M, Girardin SE, Viala J, Philpott DJ: Nods, Nalps and Naip: intracellular regulators of bacterial-induced inflammation. Cell Microbiol 2003; 5: 581-592.

13 Inohara N, Nunez G: NODs: intracellular proteins involved in inflammation and apoptosis. Nat Rev Immunol 2003; 3: 371-382.

14 Tschopp J, Martinon F, Burns K: NALPs: a novel protein family involved in inflammation. Nat Rev Mol Cell Biol 2003; 4: 95-104.

15 Dangl JL, Jones JD: Plant pathogens and integrated defence responses to infection. Nature 2001; 411: 826-833.

16 Belkhadir Y, Subramaniam R, Dangl JL: Plant disease resistance protein signaling: NBS-LRR proteins and their partners. Curr Opin Plant Biol 2004; 7: 391-399.

17 Raphael SA, Blau EB, Zhang WH, Hsu SH: Analysis of a large kindred with Blau syndrome for HLA, autoimmunity, and sarcoidosis. Am J Dis Child 1993; 147: 842-848.

18 Giachino D, van Duist MM, Regazzoni S et al: Analysis of the CARD15 variants R702W, G908R and L1007fs in Italian IBD patients. Eur J Hum Genet 2004; 12: 206-212.

19 Altschul SF, Madden TL, Schaffer AA et al: Gapped BLAST and PSIBLAST: a new generation of protein database search programs. Nucleic Acids Res 1997; 25: 3389-3402. 
20 Apweiler R, Bairoch $\mathrm{A}$, Wu $\mathrm{CH}$ et al: UniProt: the Universal Protein knowledgebase. Nucleic Acids Res 2004; 32: (database issue: D115-D119).

21 Poirot O, O'Toole E, Notredame C: Tcoffee@igs: a web server for computing, evaluating and combining multiple sequence alignments. Nucleic Acids Res 2003; 31: 3503-3506.

22 Kabsch W, Sander C: Dictionary of protein secondary structure: pattern recognition of hydrogen-bonded and geometrical features. Biopolymers 1983; 22: 2577-2637.

23 Bourne PE, Addess KJ, Bluhm WF et al: The distribution and query systems of the RCSB Protein Data Bank. Nucleic Acids Res 2004; 32: (database issue: D223-D225).

24 Albrecht M, Tosatto SC, Lengauer T, Valle G: Simple consensus procedures are effective and sufficient in secondary structure prediction. Protein Eng 2003; 16: 459-462.

25 Gouet P, Robert X, Courcelle E: ESPript/ENDscript: extracting and rendering sequence and 3D information from atomic structures of proteins. Nucleic Acids Res 2003; 31: 3320-3323.

26 Koonin EV, Aravind L: The NACHT family - a new group of predicted NTPases implicated in apoptosis and MHC transcription activation. Trends Biochem Sci 2000; 25: 223-224.

27 Leipe DD, Koonin EV, Aravind L: STAND, a class of P-loop NTPases including animal and plant regulators of programmed cell death: multiple, complex domain architectures, unusual phyletic patterns, and evolution by horizontal gene transfer. J Mol Biol 2004; 343: 1-28.

28 Bateman A, Coin L, Durbin R et al: The Pfam protein families database. Nucleic Acids Res 2004; 32: (database issue: D138D141).

29 Tameling WI, Elzinga SD, Darmin PS et al: The tomato R gene products I-2 and MI-1 are functional ATP binding proteins with ATPase activity. Plant Cell 2002; 14: 2929-2939.

30 Abrahams JP, Leslie AG, Lutter R, Walker JE: Structure at $2.8 \AA$ resolution of F1-ATPase from bovine heart mitochondria. Nature 1994; 370: 621-628.

31 Girardin SE, Hugot JP, Sansonetti PJ: Lessons from Nod2 studies: towards a link between Crohn's disease and bacterial sensing. Trends Immunol 2003; 24: 652-658.

32 Wang X, Kuivaniemi H, Bonavita G et al: CARD15 mutations in familial granulomatosis syndromes: a study of the original Blau syndrome kindred and other families with large-vessel arteritis and cranial neuropathy. Arthritis Rheum 2002; 46: $3041-3045$.

33 Hugot JP, Zouali H, Lesage S: Lessons to be learned from the NOD2 gene in Crohn's disease. Eur J Gastroenterol Hepatol 2003; 15: 593-597.
34 Martinon F, Tschopp J: Inflammatory caspases: linking an intracellular innate immune system to autoinflammatory diseases. Cell 2004; 117: 561-574.

35 Jiang X, Wang X: Cytochrome C-mediated apoptosis. Annu Rev Biochem 2004; 73: 87-106.

36 Albrecht M, Domingues FS, Schreiber S, Lengauer T: Structural localization of disease-associated sequence variations in the NACHT and LRR domains of PYPAF1 and NOD2. FEBS Lett 2003; 554: 520-528.

37 DeLaBarre B, Brunger AT: Complete structure of p97/valosincontaining protein reveals communication between nucleotide domains. Nat Struct Biol 2003; 10: 856-863.

38 Li G, Zhang XC: GTP hydrolysis mechanism of Ras-like GTPases. J Mol Biol 2004; 340: 921-932.

39 Vetter IR, Wittinghofer A: The guanine nucleotide-binding switch in three dimensions. Science 2001; 294: 1299-1304.

40 Kanazawa N, Okafuji I, Kambe N et al: Early-onset sarcoidosis and CARD15 mutations with constitutive nuclear factor- $\kappa$ B activation: common genetic etiology with Blau syndrome. Blood 2005; 105: 1195-1197.

41 Albrecht M, Lengauer T, Schreiber S: Disease-associated variants in PYPAF1 and NOD2 result in similar alterations of conserved sequence. Bioinformatics 2003; 19: 2171-2175.

42 Neven B, Callebaut I, Prieur AM et al: Molecular basis of the spectral expression of CIAS1 mutations associated with phagocytic cell-mediated autoinflammatory disorders CINCA/NOMID, MWS, and FCU. Blood 2004; 103: 2809-2815.

43 Dowds TA, Masumoto J, Zhu L, Inohara N, Nunez G: Cryopyrininduced interleukin 1beta secretion in monocytic cells: enhanced activity of disease-associated mutants and requirement for ASC. $J$ Biol Chem 2004; 279: 21924-21928.

44 Senior AE, Nadanaciva S, Weber J: The molecular mechanism of ATP synthesis by $\mathrm{F}_{1} \mathrm{~F}_{0}$-ATP synthase. Biochim Biophys Acta 2002; 1553: $188-211$

45 Antes I, Chandler D, Wang H, Oster G: The unbinding of ATP from F1-ATPase. Biophys J 2003; 85: 695-706.

46 Watanabe T, Kitani A, Murray PJ, Strober W: NOD2 is a negative regulator of Toll-like receptor 2-mediated $\mathrm{T}$ helper type 1 responses. Nat Immunol 2004; 5: 800-808.

47 Hawkins PN, Lachmann HJ, McDermott MF: Interleukin-1receptor antagonist in the Muckle-Wells syndrome. $N$ Engl J Med 2003; 348: 2583-2584.

48 Hoffman HM, Rosengren S, Boyle DL et al: Prevention of coldassociated acute inflammation in familial cold autoinflammatory syndrome by interleukin-1 receptor antagonist. Lancet 2004; 364: $1779-1785$ 